\title{
An Information Theoretic Approach for Non-rigid Image Registration Using Voxel Class Probabilities
}

\author{
E. D’Agostino, F. Maes`, D. Vandermeulen, and P. Suetens \\ Katholieke Universiteit Leuven, Faculties of Medicine and Engineering, Medical \\ Image Computing (Radiology - ESAT/PSI), University Hospital Gasthuisberg, \\ Herestraat 49, B-3000 Leuven, Belgium \\ Emiliano.DAgostino@uz.kuleuven.ac.be
}

\begin{abstract}
We propose a multimodal free-form registration algorithm that matches voxel class labels rather than image intensities. Individual voxels are displaced such as to minimize the Kullback-Leibler distance between the actual and ideal joint probability distribution of voxel class labels, which are assigned to each image individually by a previous segmentation process. We evaluate the performance of the method for intersubject brain registration with simulated deformations, using a viscous fluid model for regularization. The root mean square difference between recovered and ground truth deformations is smaller than 1 voxel.
\end{abstract}

\section{Introduction}

While maximization of mutual information (MMI) of corresponding voxel intensities 4 has been demonstrated to be highly successful for affine image registration of multimodal medical images in a variety of different applications, extending the MMI approach to non-rigid image registration is still an active area of research. While changes in the affine registration parameters affect the registration over the entire image domain and therefore in general have substantial impact on the joint intensity histogram and on the MI criterion itself, local non-rigid deformation changes typically result in only subtle histogram changes that are more difficult to assess using MI. Various approaches have been presented that differ in the way the criterion is evaluated for local non-rigid changes in the registration transformation and in the regularization that is applied to exclude non-realistic deformations and to make the non-rigid registration problem well-posed. Rueckert et al. [6] used a B-spline representation of the deformation field to impose local smoothness, whose parameters are optimized iteratively such that MI measured globally over the entire image domain is maximized. Hermosillo et al. [2] and D'Agostino et al. [1] derived the gradient of MI with respect to individual voxel displacements using a continuous and differentiable

\footnotetext{
* Frederik Maes is Postdoctoral Fellow of the Fund for Scientific Research - Flanders (FWO-Vlaanderen, Belgium).
} 
joint histogram constructed by Parzen windowing, yielding a voxel-wise force field that was used to drive free-form deformation regularized by elastic [2] or viscous fluid [1] deformation models.

However, all these approaches have so far considered MI of corresponding voxel intensities, implicitly assuming that these are related to objects that are to be aligned by registration. In fact, in the MMI criterion formulated by Maes et al. 4, image segmentation and labelling is implicit by the intensity rescaling and binning that is applied when contructing the joint intensity histogram. In this paper, we explore the possibility of non-rigid image registration by maximizing an information theoretic measure of the similarity of voxel object labels directly, rather than of voxel intensities. In our application, which is inter-subject MR brain image matching, such labels are obtained by intensity-based tissue segmentation, assigning each voxel a probability to belong to a particular tissue class. The advantage of this approach is two-fold. Firstly, the ideal correspondence of object labels in both images is known, which is not the case for the multimodal intensities themselves, which is exploited in the registration criterion as explained in Section 2. Secondly, image segmentation allows to indirectly incorporate complex object intensity models and spatial context in the registration process, which may help to guide and constrain the non-rigid matching in regions where image information by itself is ambiguous. Our long term goal is to merge image segmentation and non-rigid registration is a single framework, whereby the result of each is iteratively improved by the output of the other.

\section{Method}

\subsection{Similarity Measure}

When two monomodal images are perfectly aligned, their joint histogram becomes diagonal, such that the quality of the registration can be evaluated by off-diagonal histogram dispersion. For multimodal images however, the joint histogram at registration depends on the nature of the data itself and can in general not be predicted in advance. However, by first segmenting each image separately by assigning each voxel an object label, the multimodal image registration problem can be reduced to a monomodal one that consists of aligning identically labelled voxels (subject to appropriate regularization constraints as described below) rather than the multimodal intensities of the original images. If a unique object label is assigned to each voxel in each image (and only a small number of objects is considered), non-rigid registration of the resulting crisp label images is likely to be hampered by the lack of registration features in homogeneously labeled regions and by interpolation artefacts at object boundaries.

Instead, for the brain MR images considered in this paper, we create label images by intensity-based pixel classification using the fully automated modelbased algorithm by Van Leemput et al. [8]. Each voxel $i$ is assigned a probability $c_{i, k}$ to belong to one of four tissue classes $k$ (gray matter, white matter, CSF, and other), such that for each voxel $\sum_{k=1}^{4} c_{i, k}=1$. A $4 \times 4$ joint class distribution $p$ 
can then be constructed assuming that the probability of corresponding voxels in the two images to belong to a particular class are independent:

$$
p_{\text {actual }}=\sum_{(i, j)} P_{i j}^{T, R}=\sum_{(i, j)}\left|\begin{array}{lllll}
c_{i, 1}^{T} \cdot c_{j, 1}^{R} & c_{i, 1}^{T} \cdot c_{j, 2}^{R} & c_{i, 1}^{T} \cdot c_{j, 3}^{R} & c_{i, 1}^{T} \cdot c_{j, 4}^{R} \\
c_{i, 2}^{T} \cdot c_{j, 1}^{R} & c_{i, 2}^{T} \cdot c_{j, 2}^{R} & c_{i, 2}^{T} \cdot c_{j, 3}^{R} & c_{i, 2}^{T} \cdot c_{j, 4}^{R} \\
c_{i, 3}^{T} \cdot c_{j, 1}^{R} & c_{i, 3}^{T} \cdot c_{j, 2}^{R} & c_{i, 3}^{T} \cdot c_{j, 3}^{R} & c_{i, 3}^{T} \cdot c_{j, 4}^{R} \\
c_{i, 4}^{T} \cdot c_{j, 1}^{R} & c_{i, 4}^{T} \cdot c_{j, 2}^{R} & c_{i, 4}^{T} \cdot c_{j, 3}^{R} & c_{i, 4}^{T} \cdot c_{j, 4}^{R}
\end{array}\right|
$$

with $c_{i, k}^{T}$ and $c_{j, k}^{R}$ the class probabilities of the pairs of corresponding voxels $i$ and $j$ in the template image $T$ and reference image $R$ respectively. When the images $T$ and $R$ are properly aligned, the matrix $p$ is expected to be diagonally dominant. By considering the ideal case whereby tissue labels in $T$ and $R$ are identical and $T$ has been perfectly warped onto $R$, a joint class probability reference model can be constructed as

$$
p_{\text {ideal }}=\sum_{(i, j)} P_{i i}^{R, R}=\sum_{(i, j)}\left|\begin{array}{llllll}
c_{i, 1}^{R} \cdot c_{i, 1}^{R} & c_{i, 1}^{R} \cdot c_{i, 2}^{R} & c_{i, 1}^{R} \cdot c_{i, 3}^{R} & c_{i, 1}^{R} \cdot c_{i, 4}^{R} \\
c_{i, 2}^{R} \cdot c_{i, 1}^{R} & c_{i, 2}^{R} \cdot c_{i, 2}^{R} & c_{i, 2}^{R} \cdot c_{i, 3}^{R} & c_{i, 2}^{R} \cdot c_{i, 4}^{R} \\
c_{i, 3}^{R} \cdot c_{i, 1}^{R} & c_{i, 3}^{R} \cdot c_{i, 2}^{R} & c_{i, 3}^{R} \cdot c_{i, 3}^{R} & c_{i, 3}^{R} \cdot c_{i, 4}^{R} \\
c_{i, 4}^{R} \cdot c_{i, 1}^{R} & c_{i, 4}^{R} \cdot c_{i, 2}^{R} & c_{i, 4}^{R} \cdot c_{i, 3}^{R} & c_{i, 4}^{R} \cdot c_{i, 4}^{R}
\end{array}\right|
$$

We propose to use the Kullback-Leibler distance $D\left(p_{\text {ideal }} \| p_{\text {actual }}\right)$ between the ideal (fixed) and the actual (registration dependent) joint class probability distribution as registration measure, which needs to be minimized by optimization of the registration parameters:

$$
D\left(p_{\text {ideal }} \| p_{\text {actual }}\right)=\sum_{k} p_{\text {ideal }} \cdot \log \frac{p_{\text {ideal }}}{p_{\text {actual }}}
$$

with the sum taken over all $4 \times 4$ entries in $p_{\text {ideal }}$.

In practice, we construct $p$ by transforming voxels from $R$ into $T$, using partial volume (PV) interpolation [4] in the space of voxel labels to distribute the contribution of each sample $i$ in $R$ to the joint histogram over its 8 (in 3-D) nearest neighbours $j$ on the grid of $T$ without need for interpolation of class labels or probabilities directly:

$$
p_{\text {real }}=\sum_{i} \sum_{j} w_{i j} P_{i j}^{T, R}
$$

with $w_{i j}$ the trilinear interpolation weights and $P_{i j}^{T, R}$ as defined above. The weights $w_{i j}$ are continuous and differentiable functions of the transformation $\boldsymbol{u}(\boldsymbol{x})$ from $\boldsymbol{x}$ in image $R$ onto $\boldsymbol{x}-\boldsymbol{u}(\boldsymbol{x})$ in image $T$ [5].

\subsection{Force Field Computation}

The effect of a local displacement of a single voxel $i$ along each of the three coordinate axes on the registration criterion $D\left(p_{\text {ideal }} \| p_{\text {actual }}\right)$ can be computed by derivation of $D$ with respect to the displacement $\boldsymbol{u}_{i}=\boldsymbol{u}\left(\boldsymbol{x}_{i}\right)$, using a similar 
approach as in [5] and assuming that the number of voxels within the region of overlap of both images is constant:

$$
\begin{gathered}
\frac{\partial D}{\partial \boldsymbol{u}_{i}}=\sum_{k} \frac{\partial D}{\partial p_{\text {actual }}} \cdot \frac{\partial p_{\text {actual }}}{\partial \boldsymbol{u}_{i}}=-\sum_{k} \frac{p_{\text {ideal }}}{p_{\text {actual }}} \cdot \frac{\partial p_{\text {actual }}}{\partial \boldsymbol{u}_{i}} \\
\frac{\partial p_{\text {actual }}}{\partial \boldsymbol{u}_{i}}=\sum_{j} \frac{\partial w_{i j}}{\partial \boldsymbol{u}_{i}} \cdot P_{i j}^{T, R}
\end{gathered}
$$

Hence, a voxel-wise force field $\boldsymbol{F}(\boldsymbol{x}, \boldsymbol{u})$ can be defined that tends to displace each voxel such as to minimize $D$ :

$$
\boldsymbol{F}\left(\boldsymbol{x}_{i}, \boldsymbol{u}\right)=-\frac{\partial D}{\partial \boldsymbol{u}_{i}}=\sum_{k} \frac{p_{\text {ideal }}}{p_{\text {actual }}}\left(\sum_{j} \frac{\partial w_{i j}}{\partial \boldsymbol{u}_{i}} \cdot P_{i j}^{T, R}\right)
$$

\subsection{Viscous Fluid Regularization}

To evaluate the merits of the new registration criterion, we adopt the free-form registration approach of [1] and use the force field $\boldsymbol{F}(\boldsymbol{x}, \boldsymbol{u})$ to drive a viscous fluid regularizer by iteratively solving its Navier-Stokes governing equation:

$$
\nabla^{2} \boldsymbol{v}+\nabla(\nabla . \boldsymbol{v})+\boldsymbol{F}(\boldsymbol{x}, \boldsymbol{u})=0
$$

with $\boldsymbol{v}(\boldsymbol{x}, t)$ the deformation velocity experienced by a particle at position $\boldsymbol{x}$. An approximate solution of (8) is obtained by convolution with a Gaussian kernel $\psi$ :

$$
\boldsymbol{v}=\psi \star \boldsymbol{F}
$$

and the deformation field $\boldsymbol{u}^{(k+1)}$ at iteration $(k+1)$ is found by integration over time:

$$
\begin{gathered}
\boldsymbol{R}^{(k)}=\boldsymbol{v}^{(k)}-\sum_{i=1}^{3} v_{i}^{(k)}\left[\frac{\partial \boldsymbol{u}^{(k)}}{\partial x_{i}}\right] \\
\boldsymbol{u}^{(k+1)}=\boldsymbol{u}^{(k)}+\boldsymbol{R}^{(k)} \cdot \Delta t
\end{gathered}
$$

The time step $\Delta t$ is constrained by $\Delta t \leq \max (\|\boldsymbol{R}\|) \cdot \Delta u$, with $\Delta u$ the maximal voxel displacement that is allowed in one iteration. Regridding and template propagation are used as in [1] to preserve topology. 


\section{Results}

The method described above was implemented in Matlab, with the image resampling and histogram computation coded in C. The maximal voxel displacement $\Delta u$ at each iteration was set to 0.25 voxels and regridding was performed when the Jacobian of the deformation field became smaller than 0.5. Iterations were continued as long as the Kullback-Leibler distance $D$ decreased, with a maximal number of iterations of 180 . Computation time for matching two images of size $128 \times 128 \times 80$ is about 7 minutes on a Pentium IV $2.2 \mathrm{GHz}$ PC.

We evaluated the performance of the method and compared it with the voxel intensity based MMI method described in [1] using the same validation strategy as in 11. The method was validated on simulated images generated by the BrainWeb MR simulator [7], which were non-rigidly deformed by known deformation fields $\boldsymbol{u}^{*}$. These were generated by matching the T1-weighted BrainWeb image to real T1-weighted images of 3 periventricular leukomalacia patients, typically showing enlarged ventricles. The ground truth deformation $\boldsymbol{u}^{*}$ was generated twice, once using the voxel intensity based MMI method of [1] and once using the method described in this paper. We evaluate how well the recovered deformation $\boldsymbol{u}$, obtained by matching the original T1-weighted BrainWeb image to its deformed version using either of both methods, resembles the ground truth $\boldsymbol{u}^{*}$. Both deformations were compared by their root mean square (RMS) error $\Delta u$ evaluated in voxel units over all brain voxels $\mathcal{B}$ :

$$
\Delta T=\sqrt{\frac{1}{N_{\mathcal{B}}} \sum_{\mathcal{B}}\left(\left|\boldsymbol{u}(\boldsymbol{x})-\boldsymbol{u}^{*}(\boldsymbol{x})\right|\right)^{2}}
$$

Error values for 3 different cases are summarized in table 1 Although the RMS error is subvoxel small in all cases, the method that generated the ground truth deformation performs generally better. A typical registration result obtained with the method proposed in this paper is illustrated in figure 1, showing the template, target and matched template images and the corresponding WM and GM maps. Deformation fields obtained with both methods are shown in figure 2

Table 1. Root mean square error $\Delta u$ in voxels between ground thruth $\left(u^{*}\right)$ and recovered $(u)$ deformation fields for 3 different cases, computed either with method presented here or with the voxel intensity based MMI approach of 1 .

\begin{tabular}{|c|r|r|r|c|}
\hline$u^{*}$ & \multicolumn{2}{|c|}{ MMI } & \multicolumn{2}{c|}{ D } \\
\hline$u$ & MMI & D & MMI & D \\
\hline Case 1 & 0.42 & 0.53 & 0.54 & 0.38 \\
\hline Case 2 & 0.46 & 0.56 & 0.45 & 0.35 \\
\hline Case 3 & 0.47 & 0.46 & 0.43 & 0.33 \\
\hline
\end{tabular}



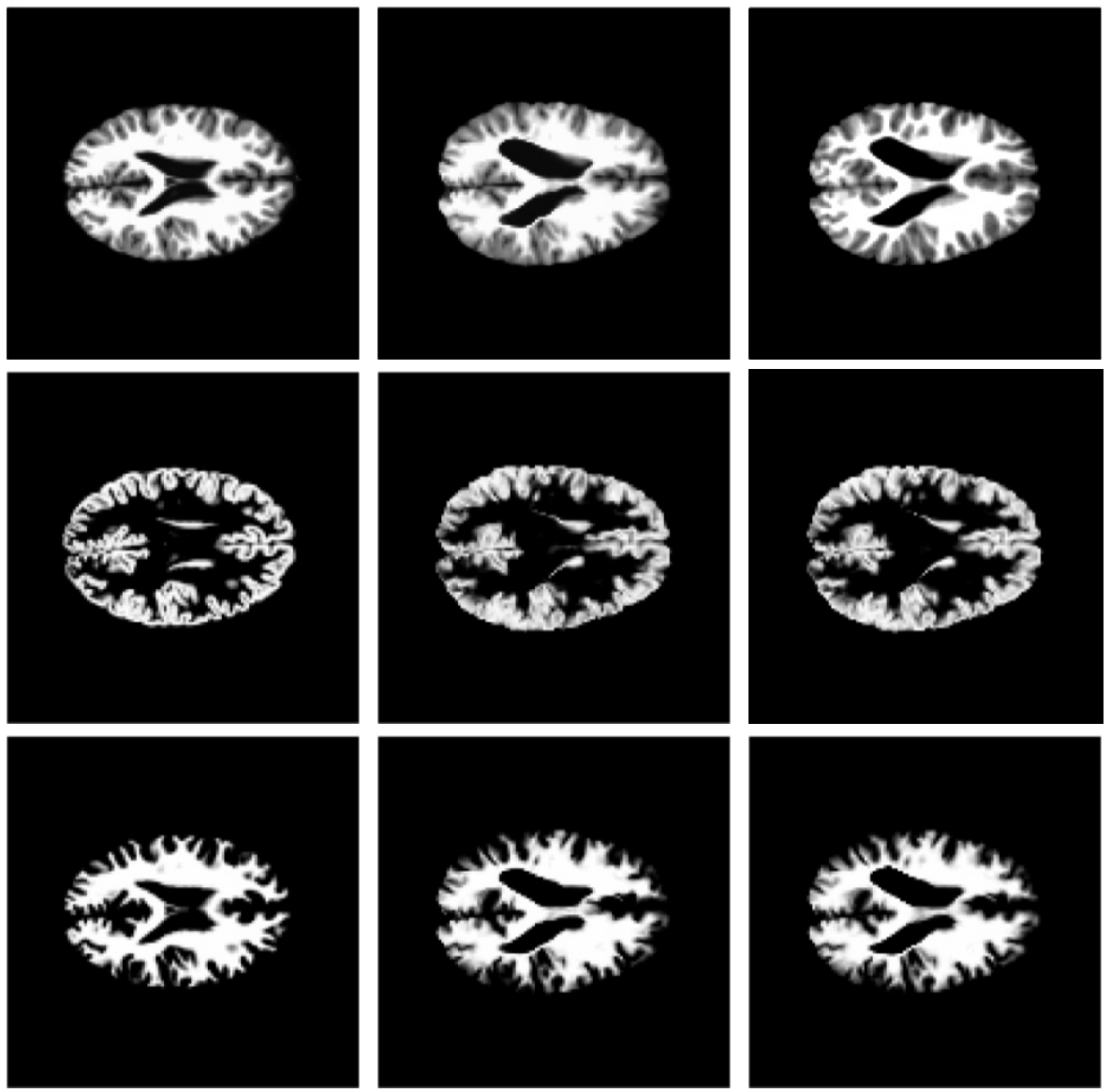

Initial:

\begin{tabular}{|l|l|l|l|}
\hline .1669 & .0926 & .0131 & .0031 \\
\hline .0509 & .2512 & .0606 & .0109 \\
\hline .0070 & .0546 & .0788 & .0135 \\
\hline .0039 & .0296 & .0434 & .1198 \\
\hline
\end{tabular}

Final:

\begin{tabular}{|l|l|l|l|}
\hline .1579 & .0634 & .0045 & .0021 \\
\hline .0637 & .2908 & .0589 & .0092 \\
\hline .0049 & .0619 & .1076 & .0196 \\
\hline .0022 & .0121 & .0249 & .1164 \\
\hline \multicolumn{4}{|c|}{ Final - Initial: } \\
\hline-.0090 & -.0292 & -.0086 & -.0010 \\
\hline .0128 & .0395 & -.0017 & -.0017 \\
\hline-.0021 & .0073 & .0288 & .0060 \\
\hline-.0017 & -.0175 & -.0185 & -.0034 \\
\hline
\end{tabular}

Model:

\begin{tabular}{|l|l|l|l|}
\hline .1586 & .0636 & .0044 & .0021 \\
\hline .0636 & .2937 & .0603 & .0105 \\
\hline .0044 & .0603 & .1093 & .0218 \\
\hline .0021 & .0105 & .0218 & .1129 \\
\hline
\end{tabular}

Fig. 1. Original intensity image and GM and WM maps for the template image (left), template image warped to reference image (middle) and reference image (right). The joint class distributions capture the coincidence of WM, GM, CSF and OTHER class voxels in the reference (columns) and template (rows) images. 

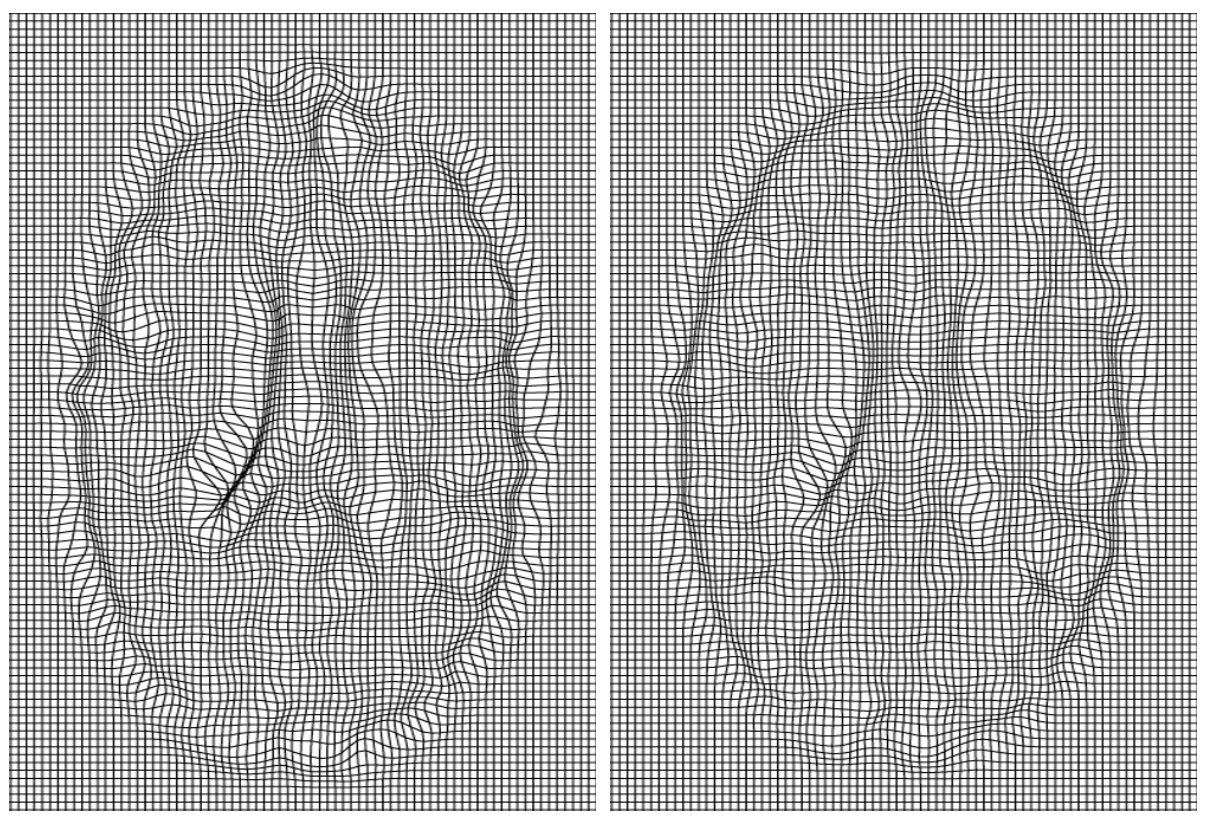

Fig. 2. Left: Deformation recovered by the MMI based method of [1]; right: deformation recovered by method proposed here.

\section{Discussion}

We present an algorithm for non-rigid image registration that optimizes an information theoretic measure of voxel class probabilities rather than voxel intensities directly. Class probabilities are assigned to each voxel by intensity-based tissue classification of each image individually and the joint class distribution is obtained by assuming that corresponding voxel class labels in each image are independent. The segmentation introduces correspondence information about the voxel features in each image, which is not available with intensity-based multimodality image registration. Hence, a good model for the joint class distribution at registration can be estimated by considering the case of perfect alignment of an image with itself. We propose to use the Kullback-Leibler distance between the observed and the model class distribution as a new registration measure. Inspection of the initial and final actual histograms $p_{\text {actual }}$ and of the model histogram $p_{\text {model }}$ depicted in figure 11, demonstrates that the joint class histogram after non-rigid matching is indeed much more similar to the model than before registration. The change in joint class probabilities prior and after registration shows that a clustering has occurred along each column, such that after registration template class labels corresponding to a particular reference class label are distributed similarly as in the model distribution.

The joint class distribution $p_{\text {actual }}$ is estimated during registration using PV interpolation [4 such that it varies smoothly with individual voxel displacements and can be analytically differentiated. A force field is thus obtained that 
acts to displace individual voxels such that the Kullback-Leibler distance between the observed and the model distribution is minimized. In the approach presented in 1, the gradient of MI is computed with respect to individual voxel displacements by modelling the joint intensity histogram as a continuous and differentiable function of the image intensity itself using a Parzen estimator. The approach presented here however is completely discrete due to the PV interpolation scheme. Also, in contrast with the method of [1, the force field (7) does not depend on the image intensity gradient of the template image, which may explain the larger smoothness of the resulting deformation field as observed in figure 2 , We demonstrated how the force field can be applied to drive free-form non-rigid registration using a viscous fluid regularization model. However, while the viscous fluid model is appropriate for inter-subject brain registration as focussed on here (whereby large deformations have to be recovered in patients showing enlarged ventricles), other regularization schemes (e.g. elastic [2]) could be used as well. Validating the performance of various regularization schemes for specific non-rigid registration applications is an important research topic and outside the scope of this paper.

A main advantage of this approach is that a priori model knowledge regarding intensity appearance or spatial context of the objects in each image can be incorporated by a model-based segmentation strategy. In our case for instance, focussing on inter-subject brain image registration, WM, GM and CSF are segmented assuming a Gaussian mixture intensity model, while also accounting for MR intensity inhomogeneity and incorporating a priori information of the expected spatial distribution of tissues in the brain [8]. Using class labels as features for non-rigid image registration opens perspectives for integrating registration and segmentation as two cooperative processes in a single framework, for instance by considering one of the images as an atlas that is non-rigidly warped onto the other and that provides a priori tissue distribution maps to guide the segmentation of the other image. Future work will be directed towards this goal.

\section{References}

[1] E. D'Agostino, F. Maes, D. Vandermeulen, and P. Suetens. A viscous fluid model for multimodal non-rigid image registration using mutual information. In Medical Image Computing and Computer-Assisted Intervention (MICCAI'02), volume 2488 of Lecture Notes in Computer Science, pages 541-548, Tokyo, Japan, September 2002. Springer-Verlag, Berlin.

[2] G. Hermosillo, C. Chef d'Hotel, and O. Faugeras. A variational approach to multimodal image matching. Technical Report 4117, INRIA-ROBOTVIS, Sophia Antipolis, France, February 2001.

[3] M.E. Leventon and W.E.L. Grimson. Multi-modal volume registration using joint intensity distribution. In Medical Image Computing and Computer-Assisted Intervention (MICCAI'98), volume 1496 of Lecture Notes in Computer Science, Cambridge, Massachusetts, October 1998. Springer-Verlag, Berlin.

[4] F. Maes, A. Collignon, D. Vandermeulen, G. Marchal, and P. Suetens. Multimodality image registration by maximization of mutual information. IEEE Transactions on Medical Imaging, 16(2):187-198, April 1997. 
[5] F. Maes, D. Vandermeulen, and P. Suetens. Comparative evaluation of multiresolution optimization strategies for multimodality image registration by maximization of mutual information. Medical Image Analysis, 3(4):373-386, 1999.

[6] D. Rueckert, L.I. Sonoda, C. Hayes, D. Hill, M.O. Leach, and D.J. Hawkes. Nonrigid registration using free-form deformations: application to breast MR images. IEEE Transactions on Medical Imaging, 18(8):712-721, 1999.

[7] BrainWeb MR simulator. Available at http://www.bic.mni.mcgill.ca/brainweb/.

[8] K. Van Leemput, F. Maes, D. Vandermeulen, and P. Suetens. Automated modelbased tissue classification of MR images of the brain. IEEE Transactions on Medical Imaging, 18(10):897-908, 1999. 